\title{
Bridging the Gap with Endoscopic (Endo luminal) Therapies for Gastro Esophageal Reflux Disease
}

\author{
John Dugan ${ }^{1,2}$, Nirav Thosani ${ }^{1,2}$, Atilla Ertan ${ }^{1,2 *}$ \\ ${ }^{1}$ McGovern Medical School, The University of Texas Health Science Center at Houston \\ ${ }^{2}$ Ertan Digestive Disease Center of Excellence, Memorial Hermann Hospital-Texas Medical Cent
}

Received: 23 March, 2016; Accepted: 12 April, 2016; Published: 17 May, 2016

*Corresponding author: Atilla Ertan, MD, FACP, AGAF, MACG, Director of the Ertan Digestive Disease Center and GI Center of Excellence, Memorial Hermann-Texas Medical Center, Professor at the University of Texas Health McGovern Medical School, Gastroenterology and Hepatology Division, phone: 713-704-5928;E-mail: atilla.ertan@uth.tmc.edu

\section{Introduction}

Gastro esophageal reflux disease (GERD) impacts the lives of over $20 \%$ of Americans, and it is one of the most common reasons for patient visits to both gastroenterologists and primary care physicians [1]. GERD affects patients' quality of life, work productivity and thus, posses a significant financial burden on community. Untreated GERD can lead to multiple complications including esophagitis, Barrett's esophagus, esophageal stricture and esophageal cancer [2].

Lifestyle modification and medical therapy including acid suppressive medications such as $\mathrm{H} 2$ blockers and proton pump inhibitor (PPI) are the first-line treatment for GERD [2]. With the invention of the PPI, GERD treatment success has significantly improved. Nonetheless, approximately $30-40 \%$ of patients are not adequately treated with medical management [3]. In addition, medication management commits patients to lifelong medications, and their potential long-term side effects, including osteoporosis, vitamin and mineral malabsorption including B12 and iron deficiency, increased infection risk, heart attacks, chronic kidney disease, dementia and others by observational studies [4-6]. These pharmacovigilance observational studies have identified associations, not causation. Yet, this has caused concern for both physicians and patients.

Laparoscopic anti reflux surgeries (LARS) have been the gold standard for surgical treatment for patients who have failed medical treatment. Less than $1 \%$ of the patients who have failed medical treatment undergo laparoscopic fundoplication. Consequently, a significant number of patients with inadequately treated reflux symptoms persist [7]. Laparoscopic fundoplication is invasive, requiring hospitalization, and carries the risk of post-op complications including dysphasia, diarrhea, and gas bloat syndrome. Approximately $50-60 \%$ of patients continue to require PPI 5 to 10 years post operation [8].

Although multiple minimally invasive endoscopic procedures including sewing, trans mural fasteners, endoscopic stapler, Enteryx (Boston Scientific, Boston, MA, USA), Gatekeeper reflux repair system (Medtronic, Inc., Minneapolis, MN, USA) have been investigated over the last two decades, and they have not been in current clinical use as a result of procedure complications and limited effectiveness [9]. Trans oral Incision less Fundoplication using the EsophyX device, radiofrequency energy treatment to lower esophageal sphincter using Stretta, and the partial anterior fundoplication using the Medigus Ultrasonic Surgical Endostapler (MUSE), are three endoscopic procedures that have been tested by prospective studies, found to be effective, approved by the United States Food and Drug Administration (FDA), and able to treat the patients that are found to be in the treatment gap of patients with chronic GERD. In this review, we will summarize the FDA approved Trans oral Incision less Fundoplication, Stretta, and muse.

\section{Trans oral Incision less Fundoplication}

Trans oral Incision less Fundoplication (TIF) using the EsophyX device is an endoscopic procedure that performs a partial (270 degree) fundoplication. It operates under the same anatomical principles of surgical fundoplication, performed endoscopically instead of surgically. The EsophyX device (Endo Gastric Solutions, Inc., Redmond, WA, USA) became available in the United States in 2007 [10]. The device and procedure has been updated over the years. The most updated procedure, described as TIF-2, came to market in 2009. The goal of the procedure is to recreate the gastro esophageal valve and thereby reduce the symptoms of gastro esophageal reflux [11].

Currently, the procedure is usually performed under general anesthesia in the outpatient setting with possible short postprocedure inpatient observation. The EsophyX device (Figure 1 , Figure 2) is placed on the endoscope. The endoscope is then inserted under direct visualization through the mouth and into the stomach. The endoscope is advanced and retroflexed to obtain direct visualization of the procedure. The tissue retractor is used to grasp the fundus, pull it into the EsophyX device, thereby recreating the gastro esophageal valve. If a hernia less than $2 \mathrm{~cm}$ is present, the endoscope and device can be advanced to reduce the hernia. After the valve is created and hiatal hernia 
(if present) reduced, the wrap is created by the tissue mold and $\mathrm{H}$-shaped polypropylene fasteners are deployed to hold the tissue in position (Figure 3). Additional fasteners are deployed to create a 2-3cm, 200-300-degree fundoplication [12-14].

Multiple studies evaluating the TIF procedure has been completed [10,15-17]. Results vary and most studies have been small with short term follow up and changes in the device and technique over time. With improvements in device and technique, the results of the procedure have also improved.

Two recent trials evaluating TIF procedure are discussed below. First, the RESPECT trial completed by Hunter and colleagues was a prospective, sham-controlled trial. One hundred seventeen patients with GERD without significant hiatal hernia $(>2 \mathrm{~cm}$ ) were randomized to sham procedure or TIF (80 patients to the TIF and 37 to the sham, PPI arm). Patients were followed over a 6-month period. TIF was ultimately found to significantly reduce $(\mathrm{P}=0.023)$ regurgitation in $67 \%$ of patients as compared to only $45 \%$ in the sham, PPI arm. In addition, esophageal $\mathrm{pH}$ improved after TIF - mean of $9.3 \%$ before TIF and $6.3 \%$ after TIF. The sham, PPI arm showed no significant improvement - mean $8.6 \%$ before sham and $8.9 \%$ after sham. The authors ultimately concluded that TIF was an effective treatment up to 6 months after the procedure for patients with refractory GERD despite PPI treatment [15].

A second recent publication, the TEMPO trial by Trad, et al. completed a 6-month multicenter randomized study comparing the efficacy of TIF against PPI in controlling GERD symptoms. Sixty patients $(39=$ TIF, $21=$ PPI $)$ participated in the study. Patients in the PPI group were started on maximum dose PPI. The other arm comprised of patients undergoing TIF with EsophyX (TIF-2). At 6 month follow up, 97\% of patients experienced elimination of regurgitation as compared to $50 \%$ in the PPI arm. In addition, $90 \%$ of patients in TIF arm had eliminated PPI use [16].

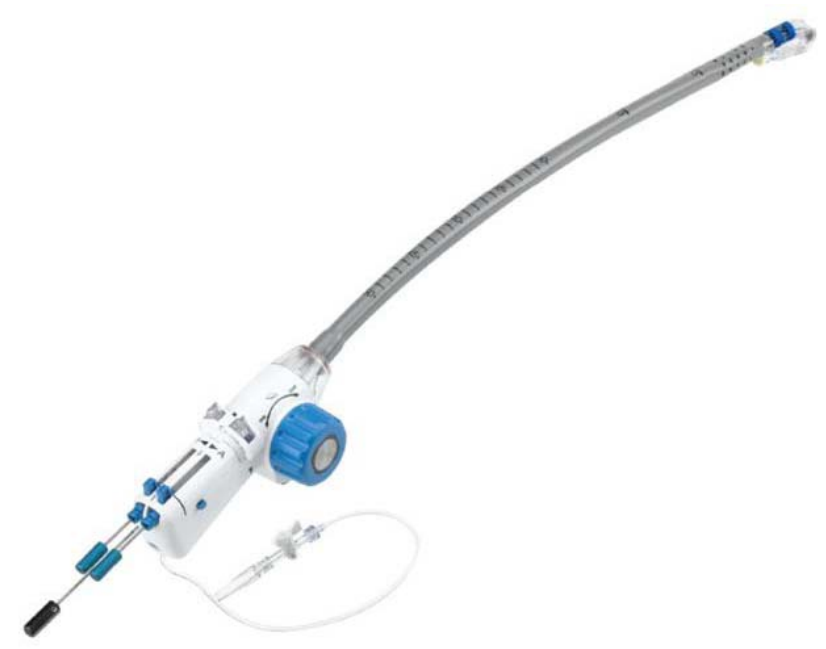

Figure 1: EsophyX device. CEndo Gastric Solutions, Inc.

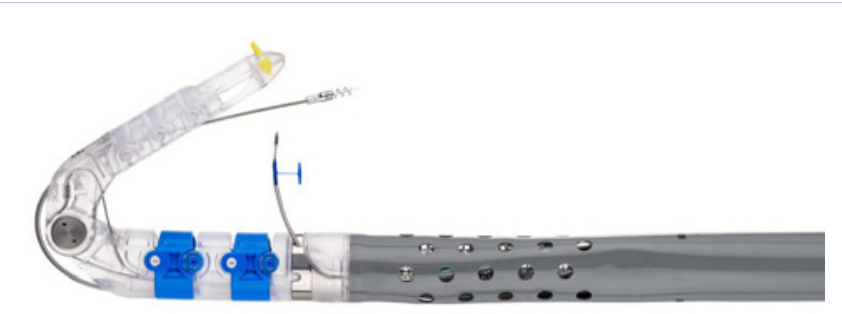

Figure 2: EsophyX device, close up. (C) Endo Gastric Solutions, Inc

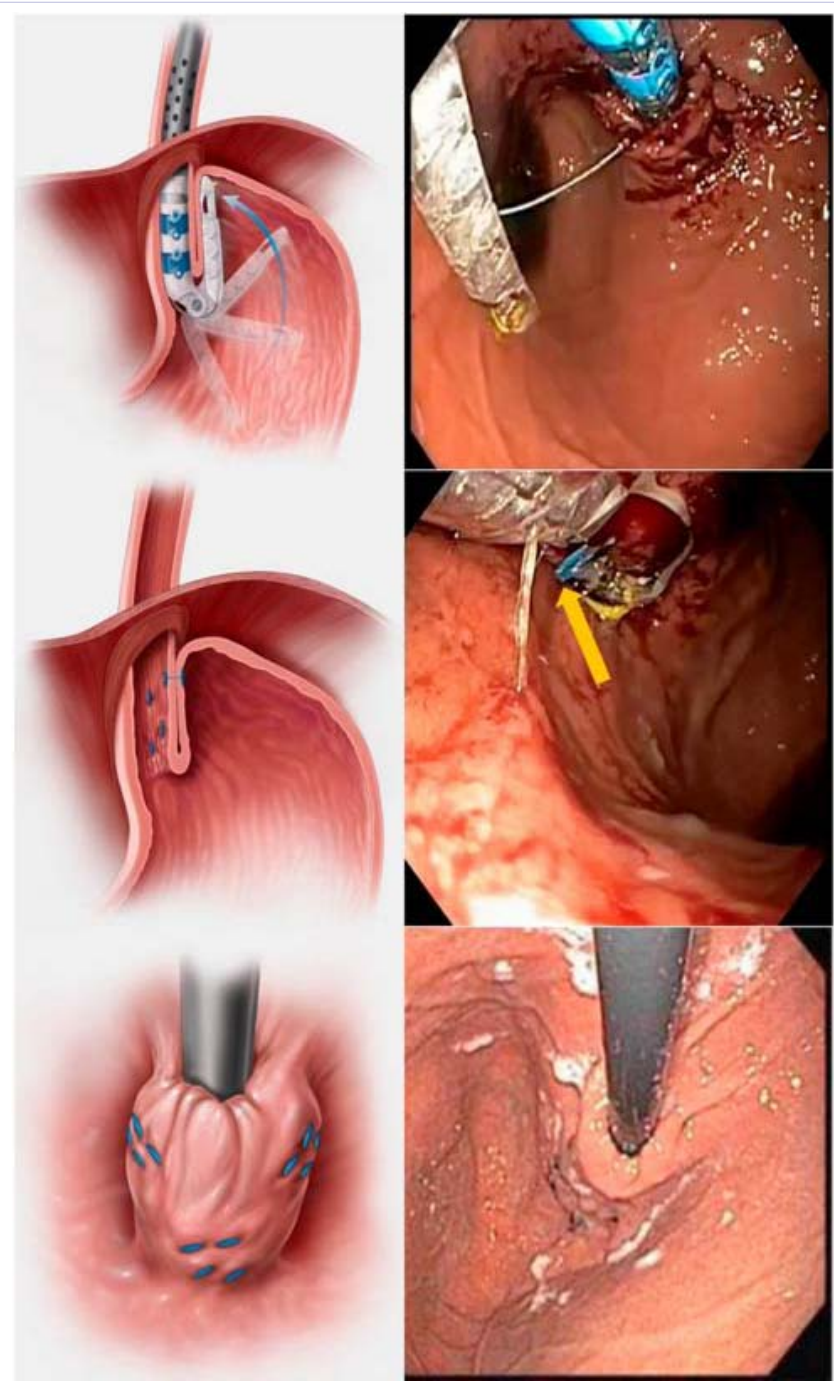

Figure 3: Summary of TIF procedure using EsophyX device. (CEndo Gastric Solutions, Inc.

As mentioned previously, most of available publications evaluating the efficacy of TIF are short-term studies. One study by Testoni, et al., evaluates the long term efficacy of TIF over a 6-year period. Fifty patients were evaluated during the study - at 6 and 12 months, 45 at 24 months, 32 at 36 months, 24 at 4 years, 
19 at 5 years and 14 at 6 years. $75-80 \%$ of patients stopped or halved their PPI use at 3 years and this remained stable up to 6 years. In addition, symptom scores were lower while off PPI. More objectively, significant improvement in impedance monitoring $(\mathrm{p}=0.01)$ was noted with fewer acid refluxes after the TIF treatment [16]. Witteman, et al also performed a study noting significant improvement in GERD symptoms scores and esophageal acid exposure at 6 months. At 12 months, symptoms scores remained improved but esophageal acid exposure no longer showed improvement [10].

In summary, TIF has emerged as a safe, effective, and durable alternative to refractory GERD patients with small hiatal hernia $(>2 \mathrm{~cm})$ without significant adverse events.

\section{Stretta}

The FDA approved Stretta (Mederi Therapeutics, Greenwich, CT, USA) in 2000. An updated clearance on the radiofrequency (RF) generator was issued 2011. The Stretta device is comprised of aRF generator and a flexible balloon catheter assembly with a four nickel-titanium needle system to deliver energy $(465 \mathrm{kHz}$, 2-5 $\mathrm{W}$ per channel, $80 \mathrm{~V}$ maximum).

The procedure is performed under sedation, usually with intravenous propofol in an outpatient setting. During the Stretta procedure, an endoscope is introduced through the mouth and into the esophagus. To obtain proper electrode placement, the distance from the incisors to the squamocolumnar junction (Z-line) is measured and a guide-wire is placed. Subsequently, the endoscope is removed and a catheter with four needleelectrodes (Figure 4) is advanced through the mouth over the guide-wire into the esophagus. Using the four needle-electrodes, radiofrequency energy is delivered to the muscularispropria with a target temperature of $85^{\circ} \mathrm{C}$ for 60 seconds. Maintaining tight temperature control prevents mucosal heat injury and stricture formation. Multiple treatments are applied by rotating the catheter circumferentially and advancing the catheter from $1 \mathrm{~cm}$ proximal to the $\mathrm{z}$-line and distally $2 \mathrm{~cm}$ to the gastric cardiac. A total of 56 treatments are applied with an average procedure time around 35-minute (Figure 5) [18-20].

The exact mechanisms of action of Stretta are not well understood. Four mechanisms have been proposed and are supported by several studies. These four mechanisms are: increased gastric yield pressure, increased thickness of the lower esophageal sphincter muscle, decreased gastro-esophageal junction compliance without fibrosis, and decreased transient esophageal sphincter relaxations. Each of these proposed mechanisms supports the ultimate goal of re-enforcing the antireflux barrier [21].

It is interesting to note that Stretta has been shown to improve gastro paresis in some patients. In 2008, Nora, et al, performed a study that showed significant improvement of gastric emptying at $30 \mathrm{~min}, 60 \mathrm{~min}$, and $90 \mathrm{~min}$ and $120 \mathrm{~min}$ intervals 6 months after the Stretta procedure. All of these patients had a 1-year symptoms follow-up assessment, which showed significant

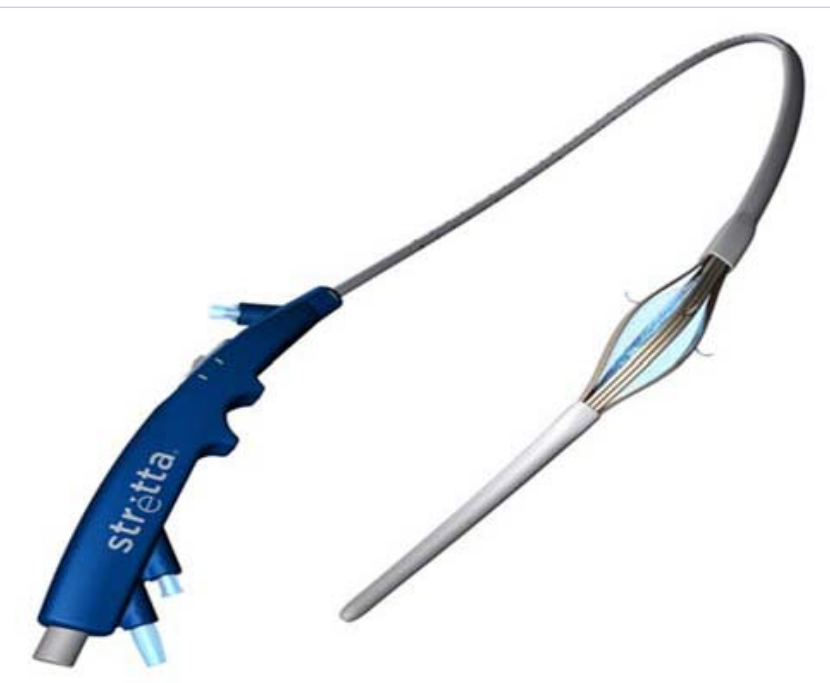

Figure 4: Stretta catheter. (C) Mederi Therapeutics, Inc.

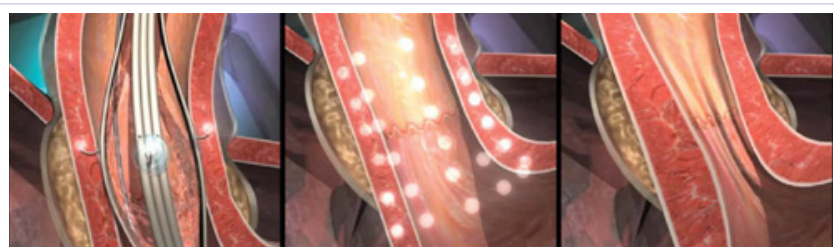

Figure 5: Stretta procedure. (C) Mederi Therapeutics, Inc.

improvements in GERD related quality of life, dyspepsia and heartburn sources [22]. However, these important findings will need to be confirmed by the other investigators.

The safety data of the procedure has been documented in several clinical trials and the over 15,000 procedures performed [23]. The most common complications observed include fever and chest discomfort, both self-limited. More serious complications of esophageal perforation and death due to aspiration pneumonia and bradyarrythmia have been described. Overall, the safety profile for the Stretta procedure is acceptable with less than 1 percent significant complications [24].

As Stretta has been available since 2000, multiple studies of variable quality, methodology and outcome measures have been completed with some conflicting outcomes. Despite these inconsistencies, Stretta has been shown to be effective in three randomized trials and over 40 other prospective clinical studies [25]. In addition, the Society of American Gastrointestinal Endoscopic Surgeons supports Stretta as an effective treatment for refractory GERD patients [26].

Two recent selected studies that shed light on Stretta will be discussed. First, Noar, et al, performed a study evaluating the 10year effect of Stretta. Notably, previous studies only demonstrated the effectiveness of Stretta up to 4 years, whereas the Noar study examined the effectiveness of Stretta up to a 10 year period. The Noar study evaluated 217 patients with a primary outcome of 
normalization of the GERD-health-related quality of life (GERDHRQL) in more than $70 \%$ of patients. Of the patients studied, $72 \%$ achieved the primary outcome. Secondary outcomes included $50 \%$ reduction/elimination of PPI use and $60 \%$ or more improvement in satisfaction at 10 years. Success was determined by achievement of at least $50 \%$ of patients. The study found that $64 \%$ of patients achieved the secondary outcomes of reduced PPI use, $41 \%$ of patients achieving complete elimination of PPI use. The study also noted a reduction in pre-existing Barrett's metaplasia from 33 patients at the onset of the study and only 5 at the conclusion of the study - an $85 \%$ reduction [27]. This important finding needs to be confirmed by other investigators.

A second publication, a double-blind randomized cross-over study of Stretta and sham treatment was completed by Arts, et al in 2012. Symptom assessment, endoscopy, manometry, 24 hour pH monitoring and a dispensability test of the gastroesophageal junction were performed at the study start and at the study conclusion three months later. Twenty-two patients were evaluated - 11 from each arm. The study determined that there were no changes in esophageal acid exposure and lower esophageal sphincter pressure at 3 months post Stretta procedure. Symptom score was significantly improved for patients undergoing the Stretta procedure. Symptom score improved from $14.7+/-1.5$ to $8.3+/-1.9$ after the initial Stretta procedure and did not significantly change after the sham procedure. In the initial sham procedure arm, the symptom score did not significantly improve after the initial sham treatment $(16.1+/-2.5$ versus $15.6+/-2.2)$ but did improve after treatment with Stretta $(16.1+/-2.5$ versus $7.2+/-1.6)$ [28].

As mentioned previously, multiple studies of variable quality, methodology and outcome measures have been completed with conflicting outcomes. A systematic review and meta-analysis was performed by Perry, et al in 2012. A total 1441 patients from 18 studies were included and determined that radiofrequency ablation to the lower esophageal sphincter does produces significant improvement on reflux symptoms [19]. On the other hand, a recent systemic review and meta-analysis evaluating 165 patients from 4 trials showed no significant evidence for efficacy of Stretta in the treatment of GERD. The study recommends the need for a large, randomized control study to conclusively address the efficacy of Stretta for the treatment of refractory GERD [29].

\section{Medigus Ultrasonic Surgical Endostapler (MUSE)}

Similar to TIF, MUSE is an endoscopic stapling system that creates an endoscopic partial fundoplication. That being said, the MUSE apparatus differs significantly when compared to the EsophyX device.

The MUSE device consists of a single use flexible surgical endostapler with video capability, ultrasonic sight with aligning screws, and stapling anvil (Figure 6). The procedure is performed by initially placing an over tube using standard technique. The MUSE device is then inserted through the over tube and into the fundus of the stomach. The endostapler is then retroflexed and pulled back until the staple cartridge is $3 \mathrm{~cm}$ proximal to the GEJ. The stomach mucosa is then clamped between the stapling cartridge and stapling anvil. Using the aligning screws and ultrasonic sight, alignment is confirmed and staples are deployed. The MUSE device is then rotated and the procedure repeated two additional times to achieve a $180^{\circ}$ anterior fundoplication (Figure 7).

The efficacy of MUSE is based upon the data from a multicenter prospective trial by Zacherl, et al. The trial evaluated 69 patients at six sites over a 6-month period who underwent fundoplication with MUSE. A total of 66 patients completed the 6-month follow up. A total of 48/66 (73\%) of patients had a greater than $50 \%$ improvement in GERD health-related quality of life (HRQL) off PPI. A total of 42/66 (64.6\%) were no longer using a PPI daily [30]. A follow up study from the initial clinical

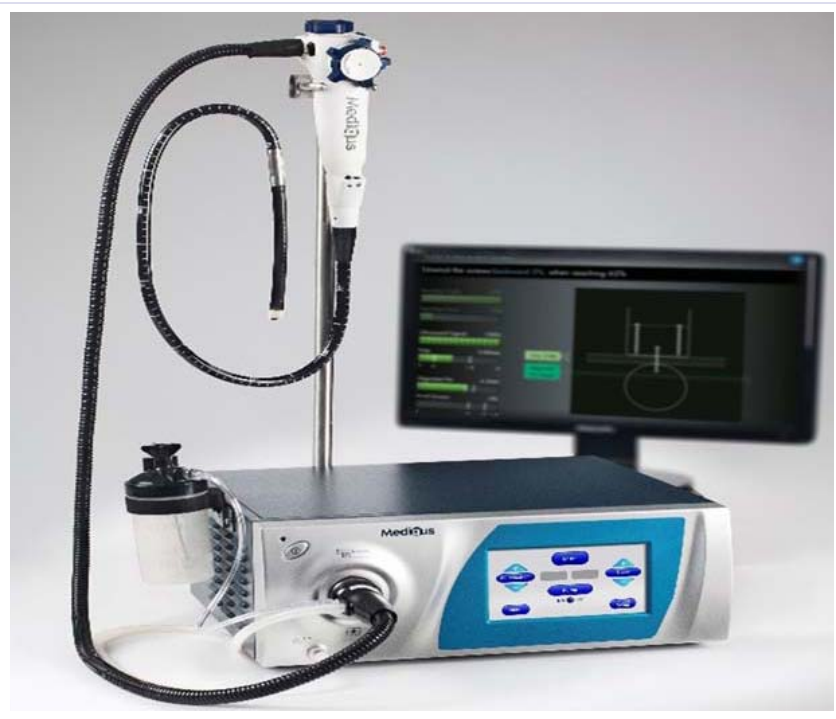

Figure 6: MUSE device. (C) Medigus, Ltd.

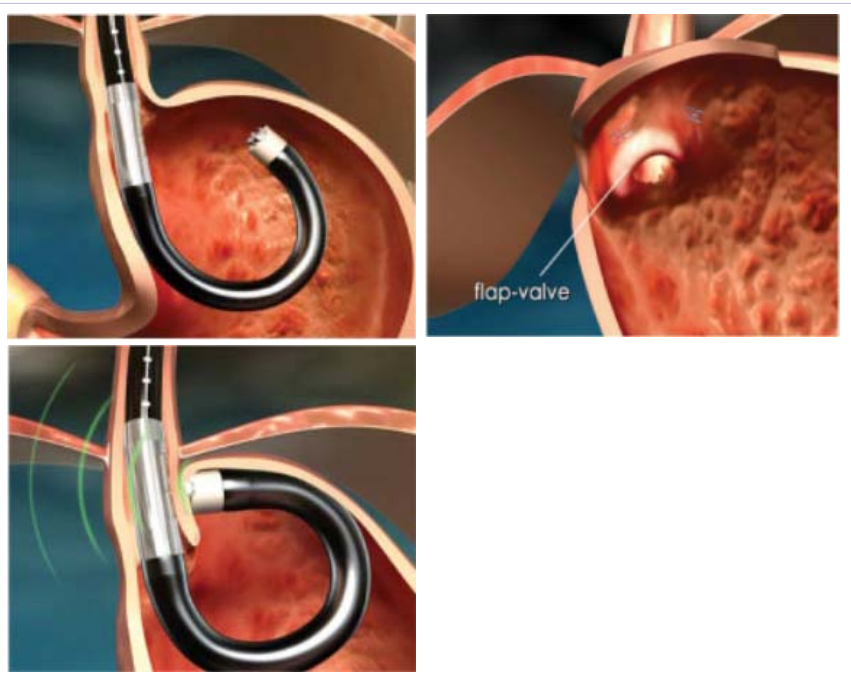

Figure 7: MUSE procedure. (C) Medigus, Ltd. 
trial was performed by Kim, et al. If evaluated 37 patients at 4 years post-procedure. A total of 25/36 (69.4\%) of patients were off PPI. In addition, the GERD-HRQL continued to be significantly decreased at 4-year follow up [31].

In the trial performed by Zacherl, et al, the most common adverse events were chest discomfort in $16 / 72$ patients $(22 \%)$ and sore throat in $15 / 72$ patients (21\%). A total of 8 serious adverse events (SAEs) were reported -4 mild, 2 moderate and 2 severe were seen in first 24 patient. The two severe SAEs included one case of empyema and pneumothorax requiring chest tube placement and hospitalization and the second case a gastrointestinal bleed requiring transfusion. The two moderate SAEs included complications of pneumomediastinum and pneumoperitoneum requiring only antibiotics, no additional intervention. Subsequent interim review of the early SAEs resulted in protocol changes and device changes. Protocol changes included increase in the number of staples deployed prophylactic therapy to reduce retching post-procedure, and a post-procedure chest X-ray to ensure no leaks are present. Device changes were made to prevent air insufflation during screw insertion to prevent pneumo peritoneum. These changes resulted in no additional cases of leak or pneumo mediastunum in the subsequent 48 patients [30].

\section{Conclusion}

In conclusion, TIF, and Stretta, and MUSE are treatment options for patients with refractory GERD. They provide long sought after minimally invasive endoscopic options to treat patients found in the treatment gap between proton pump inhibitors and fund application. MUSE appears to be safe and effective according to the initial multi-center prospective trial, but additional studies will need to be performed. Having been tested by multiple studies, TIF and Stretta have proven their effectiveness as potential treatment options that should be incorporated into the armory of gastroenterologists and selected surgeons

\section{References}

1. Kahrilas P. Gastroesophageal reflux disease. $\mathrm{N}$ Engl J Med. 2008;359:1700-1707. DOI: 10.1056/NEJMcp0804684

2. Katz P, Gerson L, Vela M. Guidelines for the diagnosis and management of gastro esophageal reflux disease. Am J Gastroenterology. 2013;108(10):308-328. doi: 10.1038/ajg.2012.444.

3. Moore M, Afaneh C, Benhori D, Antonacci C, Abelson J, Zarnegar R. Gastroesophageal reflux disease: a review of surgical decision making. World J Gastrointest Surg. 2016;8(1):77-83. doi: 10.4240/ wjgs.v8.i1.77

4. Hopkins J, Switzer N, Karmali S. Update on novel endoscopic therapies to treat gastroesophageal reflux disease: A review. World J Gastrointest Endosc. 2015;7(11):1039-1044. doi: 10.4253/wjge. v7.i11.1039.

5. Lazarus B, Chen Y, Wilson F, Sang Y, Chang AR, Coresh J, et al. Proton inhibitor use and the risk of chronic kidney disease. JAMA Intern Med. 2016;176(2):238-246. doi: 10.1001/jamainternmed.2015.7193.
6. Gomm W, Von Holt K, Thome F, Broich K, Maier W, Fink A, et al Association of proton pump inhibitos with risk of dementia - a pharmacoepidemiological claims data analysis. JAMA Neurol. 2016;73(4):410-416. doi: 10.1001.

7. Finks J, Wei Y, Birkmeyer J. The rise and fall of antireflux surgery in the United States. Surg Endosc. 2006;20(11):1698-1701.

8. Madan A, Minocha A. Despite high satisfaction, majority of gastroesophageal reflux disease patients continue to use proton pump inhibitors after antireflux surgery. Aliment Pharmacol Ther. 2006;23(5):601-605.

9. Falk G, Fennerty M, Rothstein R. AGA Institute medical position statement on the use of endoscopic therapy for gastroesophageal reflux disease. Gastroenterology. 2006;131(4):1313-1314.

10. Witteman B, Conchillo J, Rinsma N, Betzel B, Peeters A, Koek GH, et al. Randomized controlled trial of transoral incisionless fundoplication vs. proton pump inhibitors for treatment of gastroesophageal reflux disease. Am J Gastroenterol. 2015;110(4):531-542. doi: 10.1038/ ajg.2015.28

11. Cadiere G, Rajan A, Germay 0, Himpens I. Endoluminal fundoplication by transoral device for the treatment of GERD: A feasibility study. Surg Endosc. 2008;22(2):333-342.

12. Muls V, Eckardt A, Marchese $M$, , Bastens B, Buset M, Devière J, et al Three-year results of a multicenter prospective study of transoral incisionless fundoplication. Surgical Innovation. 2012;20(4):321330. doi: $10.1177 / 1553350612459275$.

13.Cadiere G, Buset M, Muls V, Rajan A, Rösch T, Eckardt AJ, et al. Antireflux transoral incisionless fundoplication using EsophyX: 12-month results of a prospective multicenter study. World J Surg. 2008;32(8):1676-1688. doi: 10.1007/s00268-008-9594-9.

14.Cadiere G, Rajan A, Rqibate M, Germay O, Dapri G, Himpens J,et al. Endoluminal fundoplication (ELF): evolution of EsophyX, a new surgical device for transoral surgery. Minim Invasive The Allied Technol. 2006;15(6):348-355.

15. Hunter J, Kahrilas P, Bell R, Wilson EB, Trad KS, Dolan, et al. Efficacy of transoral fundoplication vs omeprazole for treatment of regurgitation in randomized controlled trial. Gastroenterology. 2015;148(2):324333. doi: 10.1053/j.gastro.2014.10.009.

16. Trad K, Barnes W, Simoni G, Shughoury AB, Mavrelis PG, Raza M, et al. Transoral incisionless fundoplication effective in eliminating GERD symptoms in partial responders to proton pump inhibitor therapy at 6 months: the TEMPO randomized clinical trial. Surgical Innov. 2015;22(1):26-40. doi: 10.1177/1553350614526788.

17.Testoni P, Testoni S, Mazzoleni G, et al. Long-term efficacy of transoral incisionless fundoplication with Esophyx (Tif 2.0) and factors affecting outcomes in GERD patients followed up to 6 years: a prospective single-center study. Surg Endosc. 2015;29(1):27702780. doi: $10.1177 / 1553350614526788$.

18. Arts J, Bisschops R, Blondeau K, , Farré R, Vos R, Holvoet L, et al. A double-blind sham-controlled study of the effect of radiofrequency energy on symptoms and distensibility of the gastro-esophageal junction in GERD. Am J Gastroenterol. 2012;107(2):222-230. doi: 10.1038/ajg.2011.395

19. Perry K, Banerjee A, Melvin W. Radiofrequency energy delivery to the lower esophageal sphincter reduces esophageal acid exposure and improves GERD symptoms: A systematic review and meta-analysis. Surg Laparosc Endosc Percutan Tech. 2012;22(4):283-288. doi: 


\subsection{7/SLE.0b013e3182582e92.}

20. Utley D. The Stretta procedure: device, technique, and pre-clinical study data. Gastrointest Endosc Clin No Am. 2003;13(1):135-145

21. Triadafilopoulos G. Stretta: A valuable endoscopic treatment modality for gastroesophageal reflux disease. World Journal of Gastroenterology. 2014; 20(24):7730-7738. doi: 10.3748/wjg.v20. i24.7730

22. Noar M, Noar E. Gastroparesis associated with gastroesophageal reflux disease and corresponding reflux symptoms may be corrected by radiofrequency ablation of the cardia and esophagogastric junction. Surg Endosc . 2008;22(11):2440-2444. doi: 10.1007/s00464-0089873-4

23. Maradey-Romera C, Kale H, Fass R. Nonmedical therapeutic strategies for nonerosive reflux disease. J Clin Gastroenterol. 2014;48(7):584589. doi: 10.1097/MCG.0000000000000125.

24. Franciosa M, Triadafilopoulos, Mashimo H. Stretta radiofrequency treatment for GERD: A safe and effective modality. Gastro Research and Prac. 2013;2013:1-8. doi.org/10.1155/2013/783815

25. Muthusamy V, Lightdale J, Acosta R, et al. The role of endoscopy in the management of GERD. Gastrointest Endosc. 2015; 81(6):1305-1310. doi.org/10.1016/j.gie.2015.02.021

26. Auyang E, Carter P, Rauth T, Robert D. Fanelli. Endoluminal
Treatments for Gastroesophageal Reflux Disease (GERD). Surg Endosc. 2013; 27(8):2658-2672.

27. Noar M, Squires P, Noar E, Lee M. Long-term maintenance effect of radiofrequency energy delivery for refractory GERD: a decade later. Surg Endosc. 2014;28(8):2323-2333. doi: 10.1007/s00464-0143461-6.

28. Arts S, Bisschops R, Blondeau K, Farré R, Vos R, Holvoet L, et al. A double blind sham-controlled study of the effect of radiofrequency energy of symptoms and distensibility of the gastro-esophageal junction in GERD. Am J of Gastroenterology. 2012;107(2):222230. doi: 10.1038/ajg.2011.395

29. Lipka S, Kumar A, Richter J. No evidence for efficacy of radiofrequency ablation for treatment of gastroesophageal reflux disease: a systematic review and meta-analysis. Clin Gastroentero and Hepatol. 2015;13(6):1033-1035. doi:10.1016/j.cgh.2014.10.013.

30.Zacherl J, Roy-Shapira A, Bonavina L, Bapaye A, Kiesslich R, Schoppmann SF, et al. Endoscopic fundoplication with the Medigus Ultrasonic Surgical Endostapler (MUSE) for gastroesophageal reflux disease: 6-month results from a multi-center prospective trial. Surg Endosc. 2015;29(1):220-229. doi: 10.1007/s00464-014-3731-3733.

31. Kim H, Kwon C, Kessler W, Selzer DJ, McNulty G, Bapaye A et al. Longterm follow-up results of endoscopic treatment of gastroesophageal reflux disease with the MUSE endoscopic stapling device. Surg Endosc. 2015 